\title{
SYNAPTIC CONTACTS BETWEEN PHYSIOLOGICALLY IDENTIFIED NEURONS IN THE VISUAL SYSTEM OF THE BARNACLE ${ }^{1}$
}

\author{
BRUCE J. SCHNAPP ${ }^{2}$ and ANN E. STUART ${ }^{3}$ \\ Department of Neurobiology, Harvard Medical School, Boston, Massachusetts 02115 and Marine Biological Laboratory, \\ Woods Hole, Massachusetts 02543
}

Received January 11, 1982; Revised November 11, 1982; Accepted November 30, 1982

\begin{abstract}
Neurons in the median visual system of the barnacle were injected with horseradish peroxidase (HRP) and their processes traced in serial thick $(3 \mu \mathrm{m})$ and thin $(100 \mathrm{~nm})$ sections with light and electron microscopy. The anatomy confirms that the I-cells identified by Oertel and Stuart (Oertel, D., and A. E. Stuart (1981) J. Physiol. (Lond.) 311: 127-146) are second-order neurons in the median visual pathway. The terminal branches of the photoreceptor axons are associated in each half of the supraesophageal ganglion with a region of neuropil that extends for 30 to $40 \mu \mathrm{m}$ along the commissure; this photoreceptor-associated neuropil is continuous with but much simpler than the main mass of neuropil in the core of each hemiganglion. The photoreceptor-associated neuropil consists of a small number of different neuronal elements, each with distinctive cytological features. The photoreceptor terminal processes dominate the neuropil; in addition, one finds there the most medial processes of the arborizations of the ipsilateral and contralateral I-cells and the processes of an axon or class of axons of unknown origin referred to as the "RL" fibers. These RL processes associate and branch with the receptor axons and constitute the only major neuronal element in this region of neuropil besides the median photoreceptors and the I-cells.

In preparations with an HRP-filled I-cell, the photoreceptors were shown to be presynaptic to Icell processes at synapses which are characterized by a pair of closely apposed postsynaptic profiles referred to as a dyad. The filled I-cell constituted only one element of the dyad. Receptor endings were also presynaptic to dyads where both postsynaptic elements were unfilled. The RL processes were presynaptic to the photoreceptor terminals at nondyadic synapses, and the receptor endings made specialized junctions, possibly synaptic, with the RL processes.

Lateral to the region occupied by the photoreceptor arborization, varicosities of the I-cell are juxtaposed to processes of the A-cell; thus, these sites of the I-cell, where it is most likely to be presynaptic to the A-cell, are segregated from the sites at which it is postsynaptic to the photoreceptors. The I-cell is postsynaptic to photoreceptors and has varicosities juxtaposed to A-cell processes in both hemiganglia.
\end{abstract}

The median visual system of the giant barnacle has proved useful for studying mechanisms of sensory processing by higher order neurons (Ozawa et al., 1975, 1977; Hudspeth and Stuart, 1977; Stuart and Oertel, 1978;

\footnotetext{
${ }^{1}$ We are grateful to Torsten Wiesel for his patience, advice, and provision of facilities, to Jack McMahan and Jim Hudspeth for helpful criticism, to Robert Golder for artistic assistance, to Debbye YurgelonTodd for skilled technical assistance, and to Ruth Campbell and Sue Wilburn for typing the manuscript. We thank Jim Hudspeth for Figure 1. This research was supported by United States Public Health Service Grants EY01188 and EY03347 to A. E. S. and by National Research Service Award EY05285 to B. J. S.

${ }^{2}$ To whom correspondence should be addressed at his present address: Building 36, Room 3B 26, National Institutes of Health, Bethesda, MD 20205.

${ }^{3}$ Present address: Department of Physiology, University of North Carolina, Chapel Hill, NC 27514.
}

Oertel and Stuart, 1981). Evidence obtained from intracellular recordings suggests that the four photoreceptors (PRs) of the median eye are presynaptic to one or more pairs of decrementally conducting interneurons (I-cells) which in turn drive third-order cells (A-cells) that generate impulses (Stuart and Oertel, 1978; Oertel and Stuart, 1981). This sequence of three cell types is postulated to constitute the sensory pathway mediating the animal's reflex closure response to shadowing the median eye. As signals are passed along this chain, information on light brightening and intensity is lost while responses to dimming are enhanced; the physiological basis of this signal transformation has been elucidated (Stuart and Oertel, 1978; Oertel and Stuart, 1981).

This paper examines the anatomical organization of the neuropil in which the processes of the median PRs, I-cells, and A-cells come together, with several specific 
aims in mind. First, it is essential to have an independent test of the connectivity hypothesized from the physiological recording (Stuart and Oertel, 1978); anatomical demonstration is the only rigorous way of establishing that the interactions are monosynaptic. Second, in order to relate intracellular recordings from the I-cell body to the physiological role of this interneuron in processing the sensory information received from the PRs, it is useful to know the relative spatial distribution on the I-cell's arborization of the sites postsynaptic to the PRs and the sites presynaptic to the A-cell. In invertebrates synaptic contacts are made on processes in the neuropil and not on cell bodies; in many cases, pre- and postsynaptic sites are separated by a micron or less along the same secondary process (King, 1976; Muller and McMahan, 1976), suggesting that a very localized integration may normally take place. Finally, we hoped that an anatomical analysis of this system would provide clues as to whether other neuronal elements, as yet unrecognized by exploring the ganglion with microelectrodes, are involved in the neuronal circuit that processes the sensory information.

Previous studies of whole mounts have revealed the gross morphology of the photoreceptor and I-cell arborizations (Hudspeth and Stuart, 1977; Oertel and Stuart, 1981). In the present work we investigated the anatomy of connections among the PRs, I-cells, and A-cells by tracing processes of identified, horseradish peroxidase (HRP)-filled cells using light (LM) and electron microscopy (EM) (Muller and McMahan, 1976). Pairs of these cells were injected; their filled processes were traced at the LM level in serial thick sections, and at the EM level in serial thin sections taken from certain of the thick sections. Certain inherent difficulties with this method precluded an absolutely complete reconstruction of the neuropil; nevertheless, an informative picture of the neuropil in which these cells make contact has been obtained. This work confirms that the I-cells are second-order neurons in the median visual pathway, the processes of the PRs and I-cells forming a relatively simple neuropil in the anterior part of the ganglionic commissure. We also show that at least one other class of neuronal process is involved early in the synaptic network of the visual system, although its physiological role is at present unknown. The anatomical relationship of the synapses to one another provides a basis for interpreting the interactions among these cells observed with physiological techniques.

\section{Materials and Methods}

Giant barnacles (Balanus nubilus) from Puget Sound were supplied by David King (Friday Harbor, WA). The dissection of the median eye, ocellar nerve, and supraesophageal ganglion, the saline solutions, and the methods of electrical recording and stimulation with light have been previously described (Hudspeth and Stuart, 1977). The two antennular nerves containing the axons of the lateral eyes (Oland et al., 1983) were routinely cut in the dissection.

\section{Identification of cells and intracellular injection of $H R P$}

Recording microelectrodes were filled with $5 \% \mathrm{HRP}$ (Boehringer Mannheim) in $0.5 \mathrm{M} \mathrm{KCl}$. The resistance of each electrode was lowered from 200 to 400 megohms to 80 to 100 megohms by breaking its tip on the Sylgardcovered bottom of the recording chamber just before impaling a cell. I-cells, A-cells, and photoreceptors were penetrated under visual control in preparations superfused with oxygenated saline and were identified by their physiological responses to a 2-sec pulse of light (Stuart and Oertel, 1978). Cells were injected for 5 to $10 \mathrm{~min}$ with depolarizing, constant-current pulses (10 to $15 \mathrm{nA}$ ) of 0.5 sec duration at a rate of one per second. Injected current was measured by a virtual-ground amplifier connected to the bath.

\section{Histology for HRP preparations}

Following the injection, the HRP was allowed to diffuse for 12 to $20 \mathrm{hr}$. During this time the preparation was maintained in a large volume of iced, oxygenated saline in the dark. The preparation was then fixed and processed for HRP staining as follows. At $4^{\circ} \mathrm{C}$ the specimen was: fixed for 60 to $90 \mathrm{~min}$ in a solution of $2 \%$ glutaraldehyde, $200 \mathrm{~mm}$ cacodylate, $60 \mathrm{~mm}$ sucrose, $140 \mathrm{~mm}$ $\mathrm{NaCl}$, and $20 \mathrm{mM} \mathrm{CaCl}$; rinsed for $20 \mathrm{~min}$ with a cacodylate-buffered salt solution containing $200 \mathrm{~mm}$ cacodylate, $240 \mathrm{~mm}$ sucrose, and $140 \mathrm{~mm} \mathrm{NaCl}$; then incubated for $1 \mathrm{hr}$ in the same solution containing $0.2 \%$ diaminobenzidine (Graham and Karnovsky, 1966). The peroxidase reaction was initiated by adding $20 \mu \mathrm{l}$ of $10 \% \mathrm{H}_{2} \mathrm{O}_{2}$ to the 1-ml incubation volume. The progress of the reaction was watched under a dissecting microscope; when the injected cell became dark after 10 to $20 \mathrm{~min}$, the ganglion was rinsed with the cold, cacodylatebuffered salt solution.

To prepare the ganglion for electron microscopy, the tissue was postfixed for 1 to $2 \mathrm{hr}$ at $4^{\circ} \mathrm{C}$ with a solution of $1 \% \mathrm{OsO}_{4}, 200 \mathrm{~mm}$ cacodylate, $140 \mathrm{~mm} \mathrm{NaCl}$, and 180 $\mathrm{mm}$ sucrose. Specimens were stained en bloc for $30 \mathrm{~min}$ in $1 \%$ tannic acid (digallic acid, Mallinckrodt Inc., St. Louis, MO) in $50 \mathrm{~mm}$ cacodylate buffer (Simionescu and Simionescu, 1976), dehydrated in graded acetones, and embedded in Epon.

\section{Procedure for tracing HRP-filled cells with light and electron microscopy}

Three preparations (described below) were studied intensively at the light and electron microscopic levels; several other preparations were examined briefly. Serial, sagittal sections 3 to $4 \mu \mathrm{m}$ thick were made through the regions containing the injected cells. Each section was dried onto a glass slide previously treated with a dry lubricant (Schabtach and Parkening, 1974) to facilitate subsequent removal of the section and was photographed under water through a Zeiss $40 \mathrm{X}$, water-immersion objective. Thus, a complete map of certain portions of the marked cells in serial thick sections was available to aid in the electron microscopic analysis. Selected thick sections were remounted for thin sectioning. Serial thin sections (90 to $100 \mathrm{~nm}$ in thickness) were cut from the thick sections, mounted on Formvar-coated, single hole grids, and stained with lead citrate for $1 \mathrm{~min}$. Montages of 6 to 12 micrographs were constructed from every other section at a final magnification of $\times 20,000$.

There were two major limitations of this method of tracing processes. (1) A thin plane of tissue was damaged 
and, hence, "lost" between adjacent thick sections. (2) Within a single thin section it was sometimes impossible to distinguish a single branching process from two closely apposed processes; this problem occasionally disabled the tracing of filled processes in preparations containing two injected neurons when reaction product in the two was indistinguishable. The injection of pairs of cells rather than single neurons was, nevertheless, highly informative, as many of the branches of each cell could be followed without ambiguity and the relationship between the two cells could be established.

\section{Details of the three major preparations}

Specimen PR. A single median photoreceptor axon was filled with HRP, and its arborization in the ganglion was randomly sampled for electron microscopy. This preparation provided information on the shape of the receptor terminal branches and on the presence of profiles presynaptic to them.

Specimen PR-I. In this preparation one PR axon and one I-cell were injected. A double injection was required to visualize the more lateral regions of the $P R$ arborization and to assess its spatial relationship with the arborization of the I-cell. At the midline of this preparation (Figs. 3 and 4), the PR and the I-cell could be unambiguously distinguished; the PR enters the commissure in the median ocellar nerve, and the I-cell is recognized by its two closely apposed processes (Oertel and Stuart, 1981). The PR and I-cell processes were traced in the commissure from the mid-line toward the I-cell body by looking at the thick sections directly in the LM and by sampling them for EM. Although processes of the I-cell and the PR could not be distinguished in the LM, in the EM they could be distinguished with absolute certainty on the basis of the electron density and consistency of the HRP reaction product that filled them (Fig. 8). Identifications indicated in the light micrographs of thick sections from specimen PR-I (Figs. 3 to 5) were made by referring to electron micrographs of thin sections taken from the same thick section. It was not possible to visualize synaptic specializations in the HRP-filled PR; however, they could be seen in the unfilled PR axons which were readily followed in the serial thick sections. Beginning at a medial level, where the photoreceptors started to branch extensively, and proceeding laterally toward the region containing the PR synaptic terminals, the serial thick sections were serially thin sectioned for a distance of about $50 \mu \mathrm{m}$. Unfilled branches of PR axons (identified with absolute certainty by their continuity with one of the three unfilled axons in the median ocellar nerve) were traced in these serial thin sections to their presynaptic sites onto the HRP-filled I-cell processes. These I-cell processes, obvious because they contain a distinctive HRP-reaction product much denser than that of the injected PR, were in several cases also traced, in serial thin sections, to one of the two I-cell processes in the midline, making the identification of these elements absolute.

Specimen A-I. In this preparation an A-cell and an Icell on the same side of the ganglion were filled. In sagittal sections through the midline of the commissure (Fig. 9), the major A-cell and I-cell processes in this region could be distinguished because they are located anatomically in distinct compartments in the commissure. The primary branches of unfilled PR axons were identified by their continuity with the parent axons in the median ocellar nerve. These profiles were followed in serial thick sections and, when required, in serial thin sections taken from the thick sections, to a point about $100 \mu \mathrm{m}$ from the midline. Beginning at this level, where the PR axons start to branch extensively and form synaptic terminals, and proceeding laterally for $35 \mu \mathrm{m}$ to include about one-half of the extent of the terminal arborization, serial thin sectioning of all the thick sections and reconstruction of every other thin section into a montage of $\times 12,000$ was done as described above.

Although the small HRP-filled processes in this preparation could not be unambiguously identified, the serial analysis from the midline, $160 \mu \mathrm{m}$ laterally toward the injected cell bodies, offered a particularly clear view of the organization and neuronal composition of the commissure and the formation of the photoreceptor-associated neuropil by the neuronal elements in the commissure. Thick sections from regions more lateral than 160 $\mu \mathrm{m}$ from the midline of this ipsilateral hemiganglion, as well as thick sections from the contralateral side of the ganglion, were sampled for electron microscopy and revealed that here processes of the two injected neurons were juxtaposed.

\section{Results}

Overview of the branching pattern of median photoreceptor axons, I-cells, and A-cells. The four PRs of the median ocellus project to the supraesophageal ganglion at the midline of the commissure joining the two hemiganglia (Hudspeth and Stuart, 1977; Figs. 1 to 3). The axon of each receptor remains large (20 to $30 \mu \mathrm{m}$ in diameter) up to its entry into the commissure and then bifurcates into primary terminal processes (5 to $10 \mu \mathrm{m}$ in diameter) that are surrounded by glial sheaths and course in the commissure for a distance of 80 to $120 \mu \mathrm{m}$ toward each hemiganglion before subdividing into the terminal,

Figure 1. The supraesophageal ganglion stained with toluidine blue to show the locations and relative sizes of the ganglion cells. The two hemiganglia are joined by a commissure (COM) which itself does not contain neuronal cell bodies, but comprises fibers crossing the ganglion's midline. The cell bodies of each hemiganglion may be divided into a dorsal group of small diameter cells $(D C)$ and a ventral group of large diameter cells $(V C)$. The I- and A-cell bodies are within the dorsal group of cells. The presence of a capsule over the cells partially obscures their outlines; this thick sheath is routinely torn open for penetration of cells. Axons of the four median receptors pass in the median ocellar nerve (MON), whereas those of the three receptors of each lateral eye pass on the median aspect of each antennular nerve $(A N)$. Two of the four median photoreceptor axons are visible (arrows) in this plane of focus. Axons of the A-cells leave the ganglion through the circumesophageal connectives (CEC). Calibration bar: $400 \mu \mathrm{m}$.

Figure 2. A drawing of the commissure of the ganglion showing the schematized branching patterns of one median photoreceptor (outlined) and an I-cell (solid). The scheme is a generalization from whole mounts and serial sections of preparations containing 

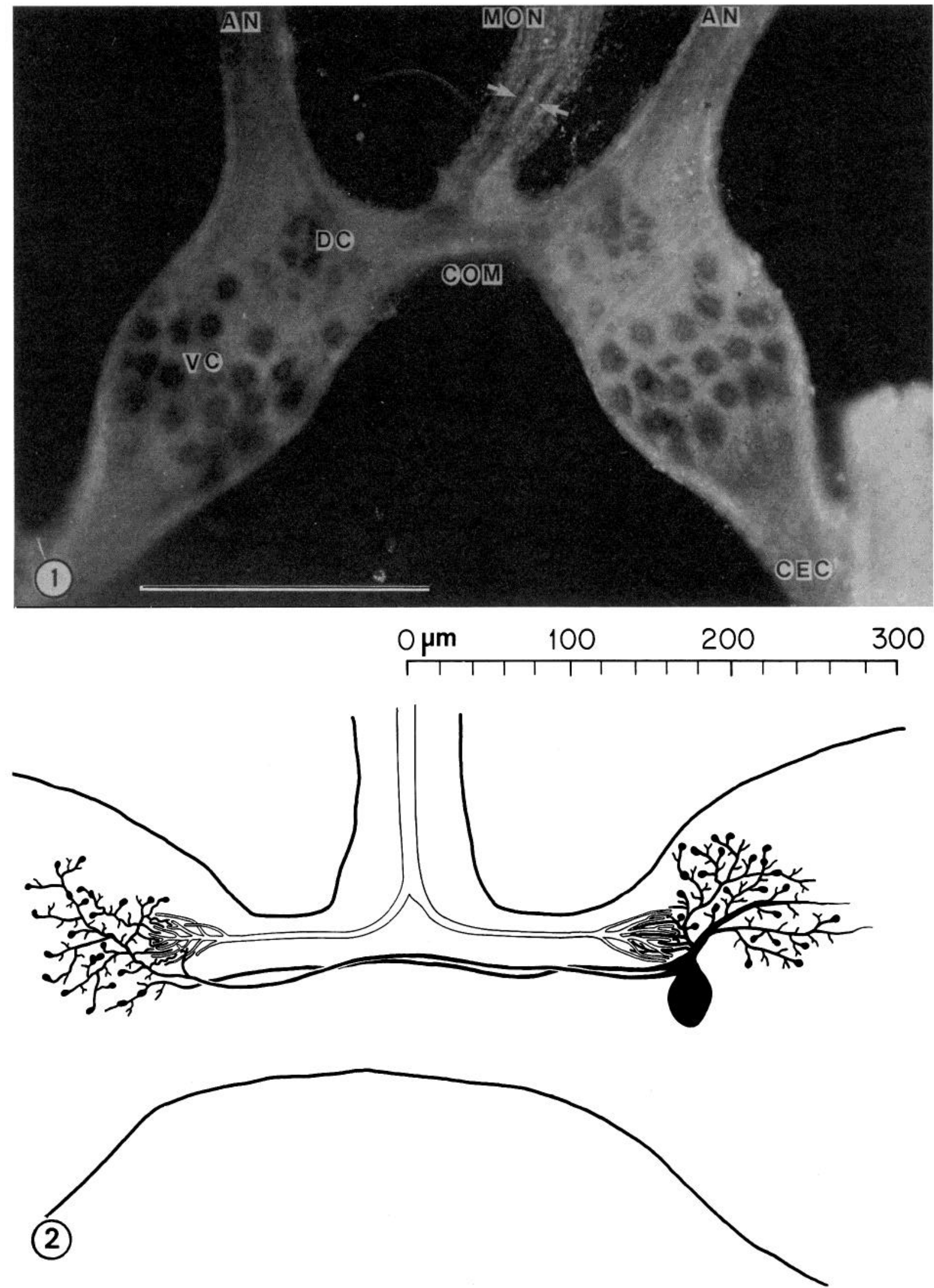

injected cells. The scale is based upon preparation $P R-I$ and is accurate in its depiction of the location from the midline of the following features: (1) the most medial photoreceptor presynaptic sites on the I-cell (135 $\mu \mathrm{m})$, (2) the most medial I-cell varicosities $(155 \mu \mathrm{m}),(3)$ the lateral border of the photoreceptor arborization $(160 \mu \mathrm{m}),(4)$ the position of the I-cell body (165 to $180 \mu \mathrm{m})$, and (5) the lateral extent of the I-cell's arborization $(280 \mu \mathrm{m})$. 

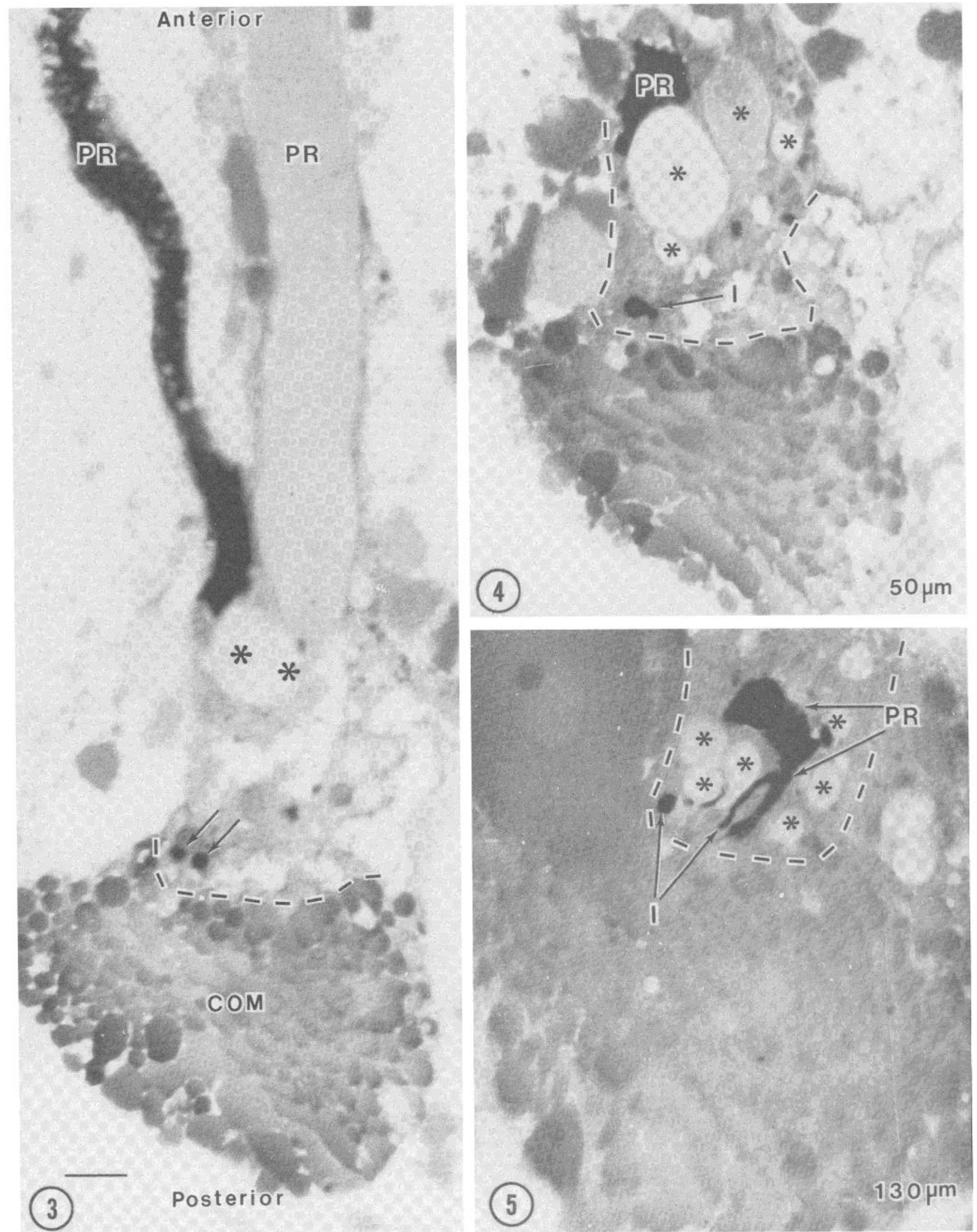

Figures 3 to 7 . Photomicrographs of $3-\mu \mathrm{m}$ sagittal sections from specimen $P R-I$ proceeding from the midline laterally toward the I-cell soma and showing arborizations of the HRP-filled and unfilled photoreceptor axons and the HRP-filled I-cell. Filled processes of the PR and I-cell were distinguished from one another by sampling thin sections from each thick section (see "Materials and Methods").

Figure 3. The series begins at the midline of the preparation, where the median ocellar nerve joins the anterior aspect (above the dotted line $)$ of the commissure $(C O M)$. Two longitudinally sectioned photoreceptors axons $(P R)$ are apparent, one of which is filled with dense HRP reaction product. The asterisks denote cross-sectioned branches of two photoreceptor axons. The two processes from the HRP-filled I-cell are indicated by arrows. The posterior compartment of the commissure (below the dotted line) contains decussating fibers. 

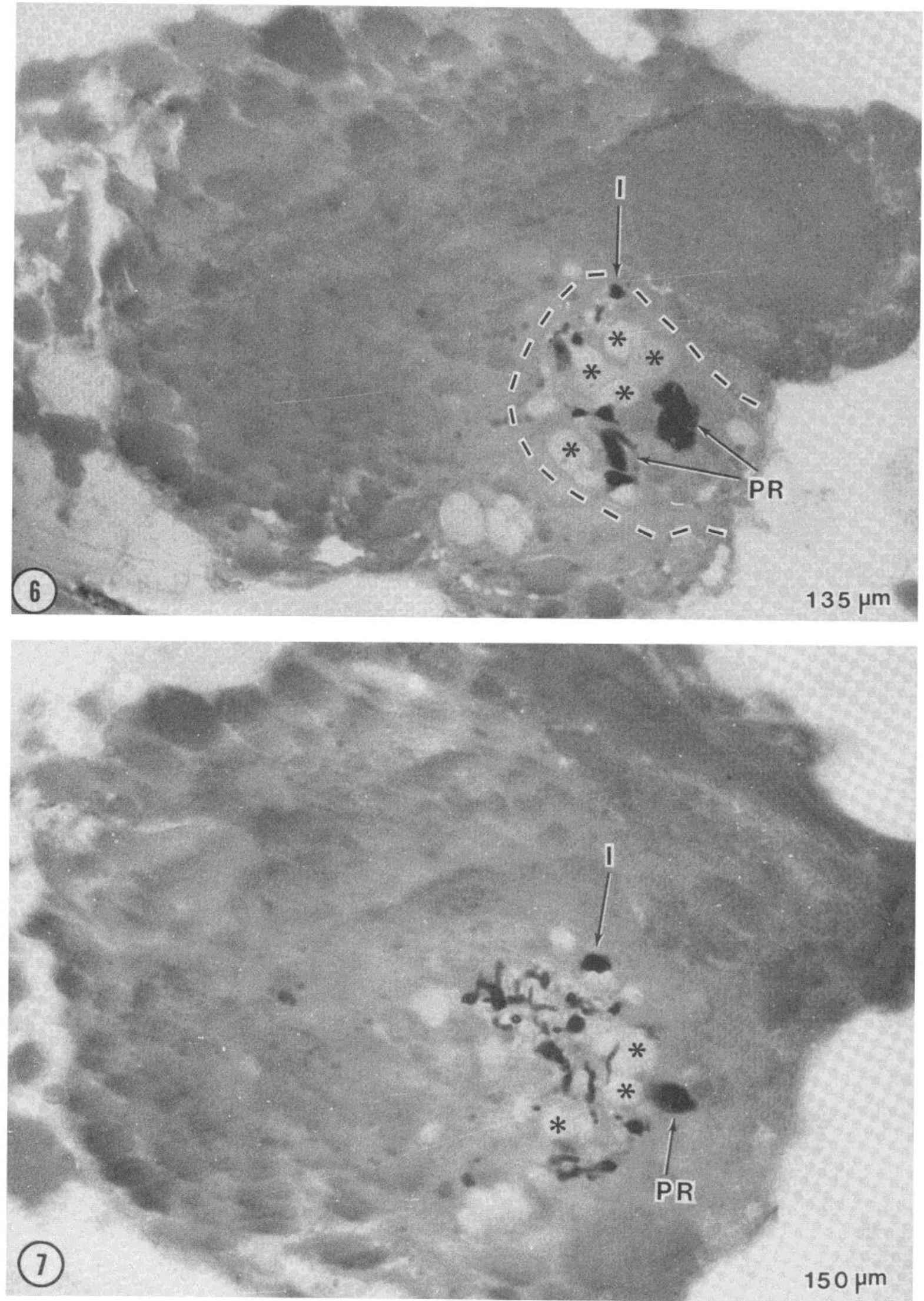

Figures 4 to 7 . The number at the bottom right of each figure indicates the approximate distance, in micrometers, of that section from the one in Figure 3. Profiles of the major branches of the unfilled photoreceptor axons (asterisks) can be identified by their translucent appearance and can be followed through the sections. Profiles of I-cell processes are labeled $I$. The receptors and the I-cell arborize together within a localized area (dotted line) separated from the posterior compartment of the commissure by glial processes evident in electron micrographs from these thick sections. As one proceeds laterally, the neuropil becomes more complex and a well defined glial border is not apparent (Fig. 7). Figures 6 and 7 have been rotated $90^{\circ}$ clockwise to Figures 3 to 5. The calibration bar in the lower left of Figure 3 corresponds to $10 \mu \mathrm{m}$ and applies to Figures 3 to 7 . 


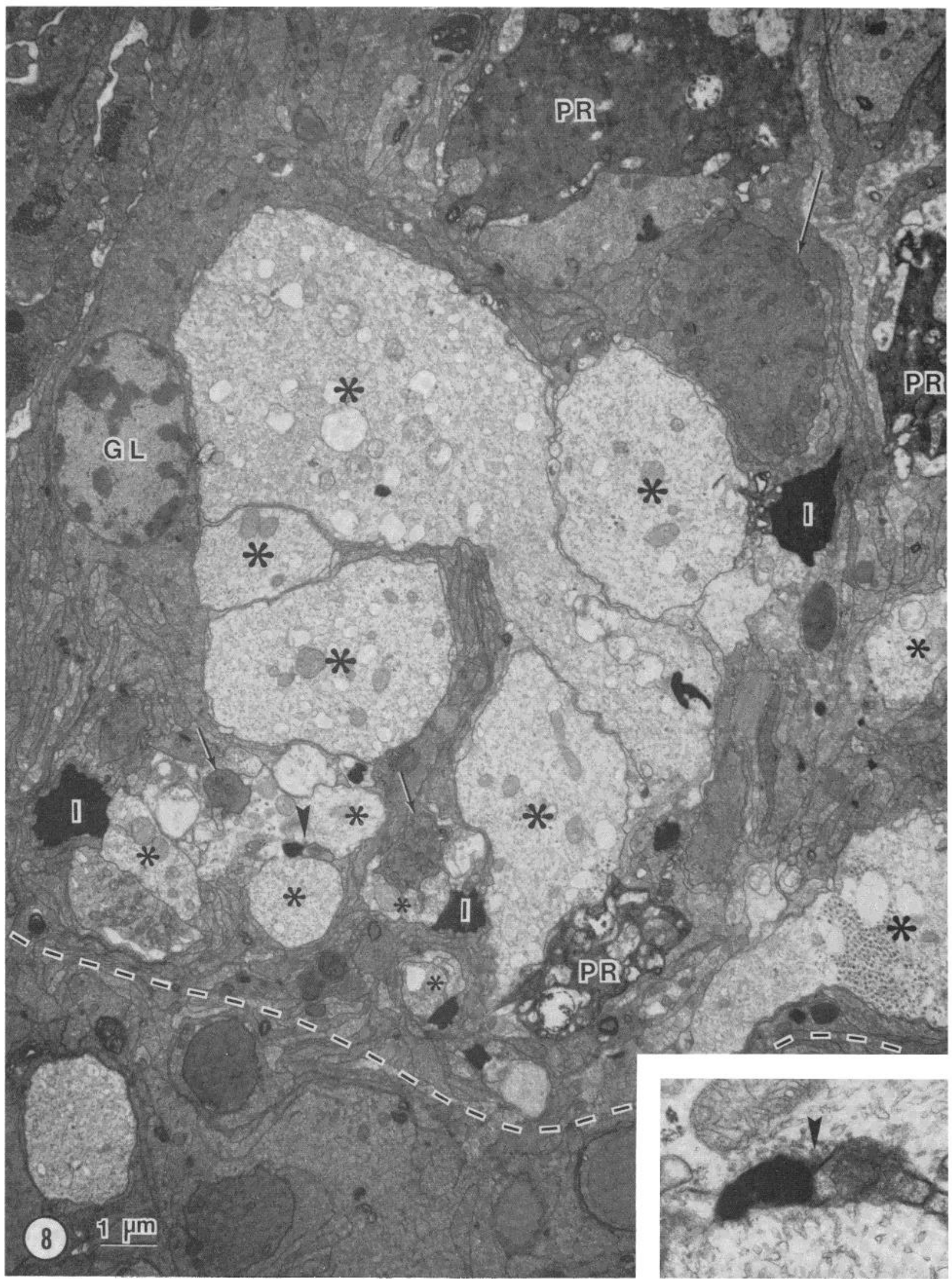

Figure 8. Thin section electron micrograph from the 3- $\mu \mathrm{m}$ section shown in Figure 6 (and rotated about $120^{\circ}$ clockwise to it) to illustrate the neuropil where the PR contacts the I-cell. The HRP-filled receptor axon branches $(P R)$ and the filled I-cell processes (three of which are labeled $I$; three smaller profiles can be seen near the bottom of the micrograph) can easily be distinguished. Branches of the unfilled receptor axons are labeled with asterisks. Note how the receptor axons cluster with each other and with the I-cell processes. A glial cell nucleus $(G L)$ is evident. The arrows point to three profiles with a dense cytoplasm 
synaptic arborization (Hudspeth and Stuart, 1977; Fig. 2 ). The primary processes of all four receptors run together in a distinct, anterior compartment of the commissure separated by layers of glial cell processes (Figs. 4,8 , and 9) from the posterior portion, which contains ganglion cell processes crossing from one hemiganglion to the other. The synaptic arborization forms within the anterior compartment of the commissure, medial to the merging of the commissure with the main mass of the hemiganglion, and extends laterally for 30 to $40 \mu \mathrm{m}$ (measured in specimens $P R-I$ and $A-I$ and indicated in Fig. 2). The photoreceptor axons can be recognized and followed for some distance in serial sections even when they are not specifically labeled (Hudspeth and Stuart, 1977).

The neurons of the supraesophageal ganglion are divided into two groups (Fig. 1): a dorsal cluster with small diameter $(20$ to $30 \mu \mathrm{m})$ somata near the commissure and a group with larger diameter (100 to $150 \mu \mathrm{m}$ ) somata capping the ventral hemiganglia (Oertel and Stuart, 1981). A. J. Hudspeth (unpublished results) counted these cells in stained whole mounts of three preparations and found an average of 43 small cells and 36 large cells per hemiganglion. The I-cell body is one of the most medially positioned somata in the cluster of small cells; it is a monopolar interneuron with two primary processes that project contralaterally, and that has arborizations overlapping with the photoreceptors' in each hemiganglion (Fig. 1 in Oertel and Stuart, 1981). Each arborization begins with a dense plexus of secondary processes. These subdivide into short tertiary branches of a diameter at or below the limit of detection in the light microscope, typically 0.1 to $0.2 \mu \mathrm{m}$ (Fig. 8) but occasionally as small as $50 \mathrm{~nm}$ (Fig. 15). Because even the largest of these tertiary processes is barely evident in whole mounts or thick sections at high magnification, their lengths have been impossible to measure by light microscopy. In serial thin sections they have been followed for over $1 \mu \mathrm{m}$. Numerous varicosities, about $2 \mu \mathrm{m}$ in diameter, are distributed along the secondary and larger tertiary processes, often attached to them by a narrow stalk. They are more numerous toward the lateral parts of the arborization and are largely absent from the most medial region, as shown diagrammatically in Figure 2.

Sagittal sections through the commissural region of specimens PR-I and A-I show that the two primary I-cell branches are present within the anterior compartment containing the receptor axons (Figs. 3, 4, and 9).

The A-cell body is usually located lateral to the I-cell body in the cluster of small cells. It sends out a single process that arborizes in each hemiganglion then leaves the contralateral hemiganglion through the connective (Ozawa et al., 1975; Oertel and Stuart, 1981). Sections of specimen A-I show that its axon runs in the posterior compartment (Figs. 9 and 10) where it is one of the largest of the commissural fibers.

The terminal branches of the PRs and the most medial secondary and tertiary processes of the I-cells intertwine in the anterior commissure, forming the medial tip of a tongue of neuropil that extends from the main neuropil of the hemiganglion toward the commissure, as described below. The A-cell's arborizations contribute to this neuropil lateral to the photoreceptor-I-cell region.

Organization of the photoreceptor-associated neuropil in the anterior compartment of the commissure. The neuropil associated with the arborization of the PRs was described in specimens PR-I and A-I by serial thick and thin sections (see "Materials and Methods"). This neuropil was found to consist of a small number of distinct neuronal components that could be traced back to primary identified processes at the midline of the commissure.

Electron microscopy of the commissural region shows that the anterior compartment is dominated by the primary processes of the photoreceptors. The primary branches are wrapped in glial sheaths; as these branches subdivide to form the terminal arborization, the sheaths end and the axonal processes become directly apposed (Figs. 12 and 13). As the PRs arborize, the relatively few other processes in the anterior commissure also branch extensively and a synaptic neuropil is formed, still obviously separated from the commissural fibers of the posterior compartment (Figs. 6 to 8). Laterally (toward the main mass of the hemiganglion) this neuropil increases in volume and complexity as neurons from the posterior compartment arborize and contribute processes (Fig. 15).

We have identified four neuronal components in the median portion of the anterior compartment of the commissure (Figs. 8 and 11). Each component has distinct cytological features that allows it to be followed laterally for about two-thirds of the extent of the PR arborization, at which point the neuropil becomes too complex to permit reliable identification of these elements. The predominant profiles are of the median photoreceptors, which have an abundant, smooth endoplasmic reticulum, large mitochondria (0.5 to $2 \mu \mathrm{m}$ in diameter), and a cytoplasmic density distinctive in a given specimen, although it can vary from one specimen to the next (Hudspeth and Stuart, 1977; Figs. 8 and 11 to 13). The second group of profiles are the fine processes of the injected Icells. A third component comprises profiles of small diameter, which tend to lie near processes of the injected I-cell (Fig. 8) and show electron-dense cytoplasm as well as the numerous small mitochondria and conspicuous aggregates of glycogen that characterize the processes of

that may be the contralateral mate of the injected I-cell (see the text). A photoreceptor-I-cell synapse (arrowhead) is shown at higher magnification in the inset. The dotted line in this and subsequent figures is drawn approximately down the center of a band of glial cell processes that separates the anterior region of neuropil from the posterior compartment of commissural fibers. Inset, Dyad synapse between a median receptor terminal and a filled process belonging to the injected I-cell. The other element of the dyad is unfilled. The filled postsynaptic process could be traced to its juncture with the large process of the I-cell shown on the left of the figure and from there to one of the two major processes of this cell seen in Figures 3 and 4 . The PR profile was traced in serial thin sections to a large primary branch and thence in thick sections to an axon at the midline. Arrowhead indicates the presynaptic density in the receptor terminal. $\times 29,000$. 

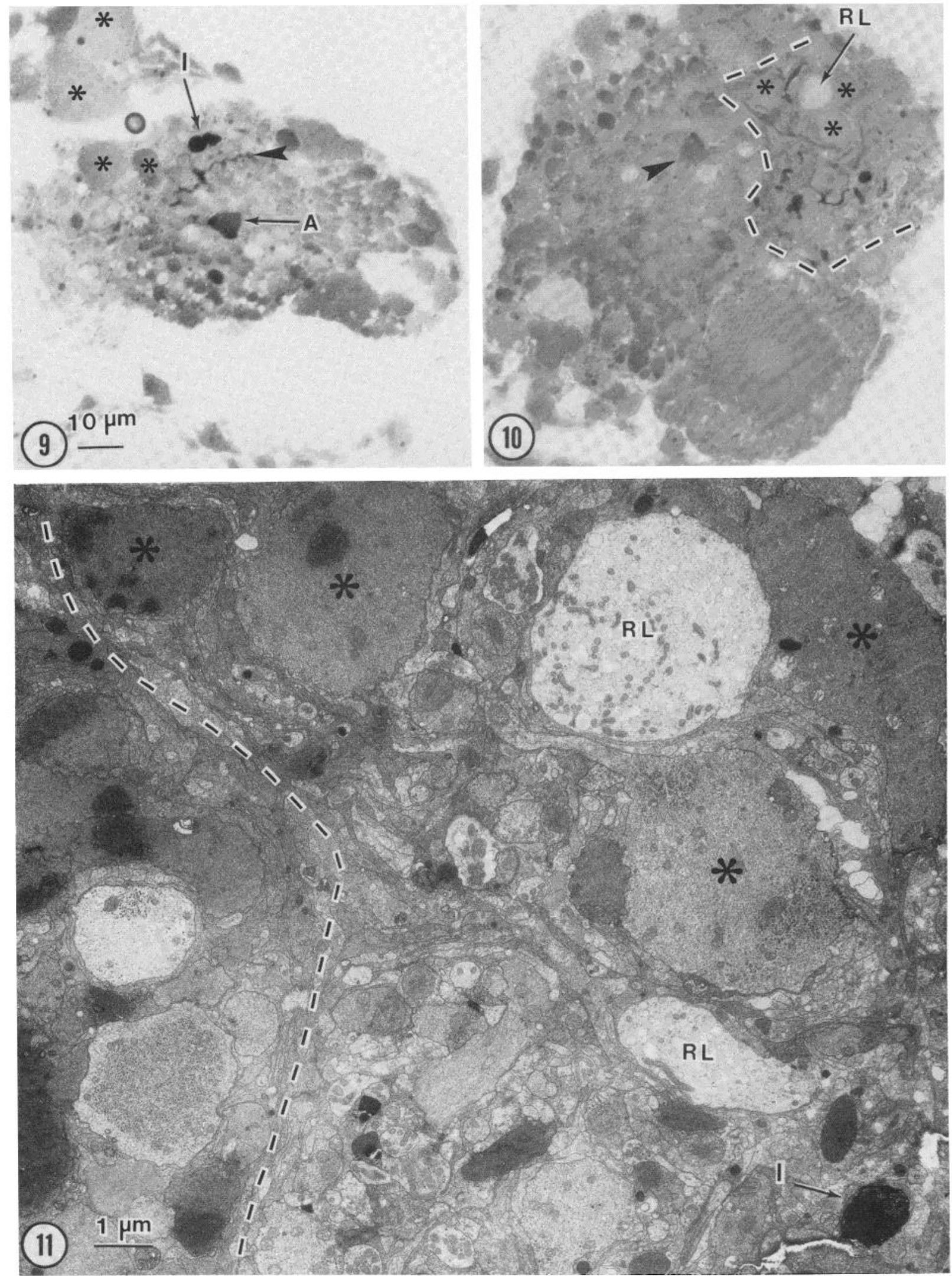

Figures 9 and 10. Photomicrographs of 3- $\mu \mathrm{m}$ sagittal sections from specimen $A-I$, proceeding laterally toward the A-cell and Icell bodies to show the relationship of the I-cell and A-cell to one another and the appearance of the RL profiles. The two figures were printed at the same magnification and in the same orientation.

Figure 9. A section within $30 \mu \mathrm{m}$ of the midline shows the prominent primary branches of the four photoreceptor axons (asterisks) and the two I-cell processes $(I)$ in the anterior compartment of the commissure, and the A-cell $(A)$ process, one of the 
the filled I-cells (Fig. 15). Their size, juxtaposition with filled I-cell processes, and cytoplasmic inclusions suggest that these profiles are those of the contralateral mate of the filled I-cell.

In specimen A-I a fourth type of profile was found in the photoreceptor-associated neuropil whose size, shape, and branching pattern are virtually identical to that of the PRs, yet its cytological features are very different. We refer to these profiles as "RL," or "receptor-like" elements (Figs. 10 to 13). They have a light cytoplasm, and their mitochondria are smaller and more numerous than those of the median receptors. Their terminals have large $(65$ to $80 \mathrm{~nm}$ ), densely filled vesicles and many round clear ones (about $40 \mathrm{~nm}$ ). The RL profiles were traced backward in specimen $A-I$ to one or more large axons which entered the commissure from outside the connective tissue surrounding the ganglion about $130 \mu \mathrm{m}$ lateral to its juncture with the median ocellar nerve, as indicated schematically in Figure 16. The axons at once became situated with the median photoreceptors (Figs. 10 and 11) and branched laterally with them (Figs. 12 and 13). In specimen $P R$, profiles with similar cytoplasmic structure were observed (Fig. 14) to be presynaptic to the filled PR. These profiles were not traced.

Synaptic contacts among PRs, I-cells, and RL profiles. Within the photoreceptor-associated neuropil, the branches of the receptor axons, the I-cells, and the RL processes come together in clusters distinctive of this region of the neuropil (Figs. 8 and 12 to 14). In these clusters, synaptic contacts are made between PRs and Icells (Fig. 8), as well as between PRs and the RL profiles (Figs. 12 to 14). The schematic diagram inset to Figure 16 summarizes the contacts among these cells as seen in preparation $A-I$.

Hudspeth and Stuart (1977) showed that the median photoreceptors contact a pair of postsynaptic processes, forming a dyadic synapse. This synapse is characterized by a V-shaped presynaptic protrusion between the two postsynaptic processes. A presynaptic density is situated at the apex of the $V$ and is associated with clear synaptic vesicles, approximately $60 \mathrm{~nm}$ in diameter. In specimen $P R-I$, dyadic synapses could be recognized on the basis of the presynaptic protrusion and its density; filled I-cell processes were found to contribute one of the postsynaptic profiles at these synapses (Fig. 8, inset). Synaptic vesicles were reduced in number, much smaller in diameter $(20$ to $30 \mathrm{~nm})$, and more pleomorphic than those described by Hudspeth and Stuart (1977). The terminals contained elongated membrane cisternae not described by the previous authors. It is not surprising that the synapses in these preparations, which were subjected to physiological recording and then not fixed until 12 to 20 $\mathrm{hr}$ after dissection, show ultrastructural changes in membranous compartments that are known to be sensitive to neuronal activity (Heuser and Reese, 1973; Schaeffer and Raviola, 1978).

In specimen $P R-I$, postsynaptic dyads consisted of one HRP-filled and one unfilled profile or two unfilled profiles, but we never observed two filled profiles. Filled postsynaptic profiles were evident on both sides of the ganglion, indicating that the I-cell is postsynaptic to the PRs at both its ipsilateral and contralateral arborizations. Contacts between PRs and HRP-filled processes were also observed in specimen $A-I$ (Fig. 12), but here the identity of the filled profiles was uncertain. Secondary branches of the PRs made synaptic contacts onto the Icell processes as close as $5 \mu \mathrm{m}$ from the points at which the secondaries branched from the primary processes in the commissure.

Specialized contacts were found between the RL processes and those of the median photoreceptors in specimen $A-I$ (Fig. 13). Figure 14 shows examples of synapses, apparently nondyadic, from RL profiles onto the injected photoreceptor (specimen $P R$ ). Here a striking presynaptic density is surrounded by small, clear vesicles; the larger dense-cored vesicles are some distance away. Similar synapses were seen in specimen $A-I$ (Fig. 13). A second type of contact between receptors and RL profiles (Fig. 12) is characterized by a density in the receptor, but because synaptic vesicles are not clearly associated with this density and because these contacts are not dyadic, it is not certain whether these junctions are synaptic or adhesive.

Possible presynaptic sites of the I-cell. Sites having the characteristic shape of the dyadic synapse were found in both the ipsilateral and contralateral arborizations of the I-cell, always within varicosities (Fig. 15) located either along the secondary branches or on the ends of small tertiary processes (diagrammed in Fig. 16). It was unfortunate that the HRP-reaction product always obscured any presynaptic specializations at these sites, making positive identification of synapses impossible.

In both of the specimens in which the I-cell had been injected ( $P R-I$ and $A-I)$, sites where the I-cell is postsynaptic to the PRs are almost completely segregated from its putative presynaptic sites. The mcst medial of the Icell's varicosities overlaps with only the most lateral 5 to $10 \mu \mathrm{m}$ of the receptor terminals (diagrammed in Figs. 2

largest commissural fibers in the posterior compartment. An unintentionally stained glial cell (arrowhead) is evident at the boundary between the anterior and posterior compartments. The filled neuronal processes were identified by tracing them in serial sections; other dense profiles in the field are not filled with HRP. The glial cell could be distinguished from the filled neurons in thin sections because the HRP was located only within membrane-bound vesicles inside this cell.

Figure 10. A section approximately $100 \mu \mathrm{m}$ lateral to that in Figure 9 and $40 \mu \mathrm{m}$ medial to the beginning of the photoreceptor terminal arborization shows a large RL profile $(R L)$ situated among the primary branches of three median receptor axons (asterisks). The fourth PR axons has subdivided medial to this section. The filled A-cell process (arrowhead) appears less dense in this thick section.

Figure 11. A thin section, taken from the thick section shown in Figure 10 and oriented similarly, illustrates the difference in appearance of $\mathrm{PR}$ and $\mathrm{RL}$ profiles. RL profiles $(R L)$ have a much lighter cytoplasm than the PRs (asterisks) and swollen axoplasmic reticulum. One filled I-cell process (arrow) could be traced in serial sections to one of the two major I-cell processes in Figure 9. 

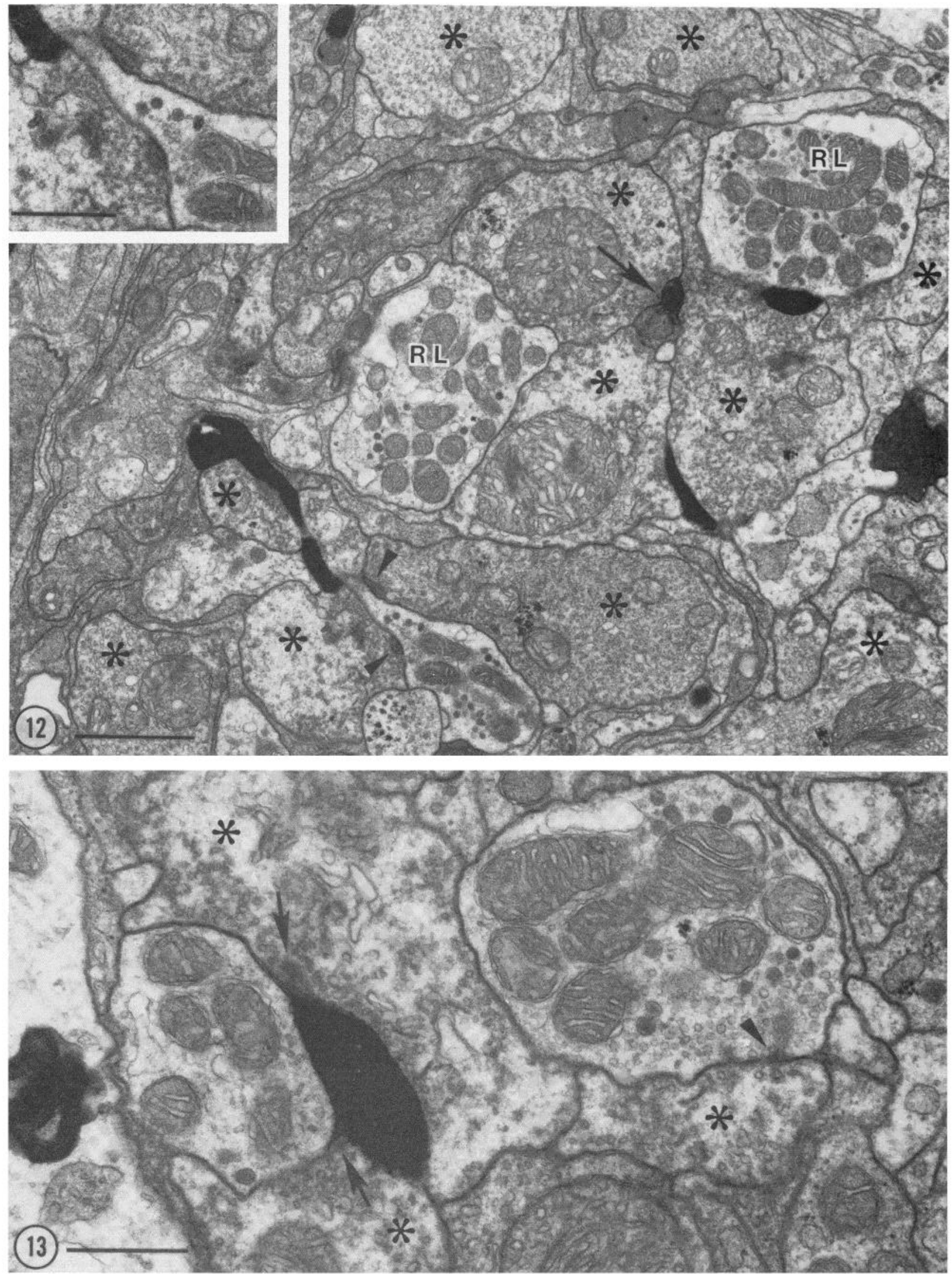

Figures 11 to 13 . Thin sections through the photoreceptor arborizations to show the relationship between the PR, RL, and Icell profiles. 
and 16). In the varicose region of its arborization, the Icell is also postsynaptic to a variety of unidentified terminals. The neuropil in this region is much more complex than it is in the photoreceptor-associated region, where the I-cell varicosities are not present and the I-cell is probably only postsynaptic.

Region of contacts between the I-cell and the A-cell. Contacts between filled I-cell and A-cell processes were found on both the ipsilateral and contralateral sides of the ganglion in specimen $A-I$. The contacts are confined to regions lateral to the $\mathrm{PR}$ arborization.

Figure 15 provides two examples of juxtapositions of Icell and A-cell processes. The contact on the right has a classical dyadic configuration with the I-cell forming the presynaptic element. Numerous examples of direct contacts between filled processes were found; these contacts were likely to be synaptic because of the characteristic $\mathrm{V}$-shaped protrusion of the presynaptic cell between two postsynaptic processes, but the masking of the presynaptic organelles by the HRP reaction product precluded their definite identification as such.

\section{Discussion}

In this report we show that the barnacle's median photoreceptors are presynaptic to I-cells, which in turn contact A-cells; this provides evidence in support of the sequence of connections proposed by Stuart and Oertel (1978) and Oertel and Stuart (1981) based on intracellular recording. The region of neuropil where the I-cell is contacted by PRs is relatively simple, permitting the tracing of the initial synaptic connections along this visual pathway. Our anatomical observations provide additional points of interest, particularly for interpreting the physiological responses of these cells: (1) the ipsilateral and contralateral arborizations of an I-cell appear to be anatomically equivalent, not presynaptic in one hemiganglion and postsynaptic in the other; $(2)$ the sites where an I-cell is postsynaptic to the PRs are segregated from the sites at which it contacts the A-cell; (3) PRs contact, and are contacted by, an apparently homogeneous class of neuronal profiles which are as yet unidentified physiologically.

The PR axons have a synaptic arborization extending for 30 to $40 \mu \mathrm{m}$ on each side of the commissure. Each arborization defines a region of neuropil consisting of a small number of distinct neuronal elements. In addition to the PRs there are: large RL profiles whose branching pattern resembles that of the median photoreceptors; the injected I-cell (in specimens in which the I-cell had been injected); and a class of processes that could reasonably be attributed to the contralateral uninjected I-cell.
The RL profiles could be traced in specimen A-I to an axon or axons with an anterior entry point in the commissure. The antennular nerve, containing the axons of the three lateral photoreceptors (Oland et al., 1983) but also many other fibers, is the only nerve entering this region. It is, therefore, reasonable to suppose that the RL processes are those of the lateral photoreceptors. If this is the case, it is surprising that the ultrastructure of the $\mathrm{RL}$ and of the median photoreceptor profiles is so different, because we have as yet detected no differences in their physiology (Oland et al., 1983). Perhaps the different ultrastructures reflect the treatment of the preparation before fixation: The median photoreceptors were intact and continued to function throughout the HRP injection and subsequent period of diffusion, whereas the axons of the lateral photoreceptors were cut during the dissection. A similar explanation may be offered for the differences in observations on the median receptors reported by us and by Hudspeth and Stuart (1977). These authors reported large glycogen deposits and dense core vesicles in the median receptor terminals, which we did not see in the three preparations described here.

From previous physiological observations there has been no way to judge whether the two arborizations of the I-cell were equivalent or whether one was "dendritic" and the other "axonal." The present results show that the PRs contact an I-cell in both hemiganglia; likewise, an I-cell contacts an A-cell in both hemiganglia. Physiological experiments have shown that an I-cell can drive both A-cells (Oertel and Stuart, 1981), which, taken together with the anatomy, implies that an I-cell contacts at least both arborizations of both A-cells. Preliminary physiological results suggest that it contacts other thirdorder cells as well (A. E. Stuart, unpublished observations).

The I-cell appears to have its presynaptic sites within varicosities and its postsynaptic sites on fine, more medial secondary or tertiary branches. The most medial 30 to $40 \mu \mathrm{m}$ of its arborization are postsynaptic to the PRs but are virtually devoid of varicosities. For the lateral 100 to $120 \mu \mathrm{m}$ of its arborization, the I-cell has pre- and postsynaptic sites interspersed along its branches. Thus, for at least two-thirds of its arborization, the I-cell conforms to the basic pattern for other invertebrate neurons (King, 1976; Muller and McMahan, 1976) in which preand postsynaptic sites occur along the same branches in the neuropil. As Muller and McMahan (1976) point out, the close association of pre- and postsynaptic sites on a cell's secondary processes could be interpreted to mean that small, localized regions of the neuropil represent discrete integrative units which act semi-autonomously.

Figure 12. A section from the medial half of the photoreceptor arborization shows specialized junctions between $\mathrm{M}$ and RL profiles (arrowheads). The terminal branches of the median photoreceptors (some labeled with asterisks) and the RL fibers are directly apposed to each other. Intercalated between them are small, HRP-filled profiles and some unfilled profiles of a similar size which form the elements of postsynaptic dyads (arrow). The unfilled profiles were identified by their distinctive cytological features. Calibration bar indicates $1 \mu \mathrm{m}$. Inset, Higher magnification of the two PR-RL junctions shown in the lower half of the figure. Calibration bar indicates $0.5 \mu \mathrm{m}$.

Figure 13. An RL profile, distinguished in this section by its dense core vesicles, appears to make a contact with a median PR terminal (arrowhead). A presynaptic density extends into the cytoplasm and is surrounded by vesicles. Synapses (arrows) are also seen between median receptor terminals (asterisks) and dyad having one element filled with HRP. 


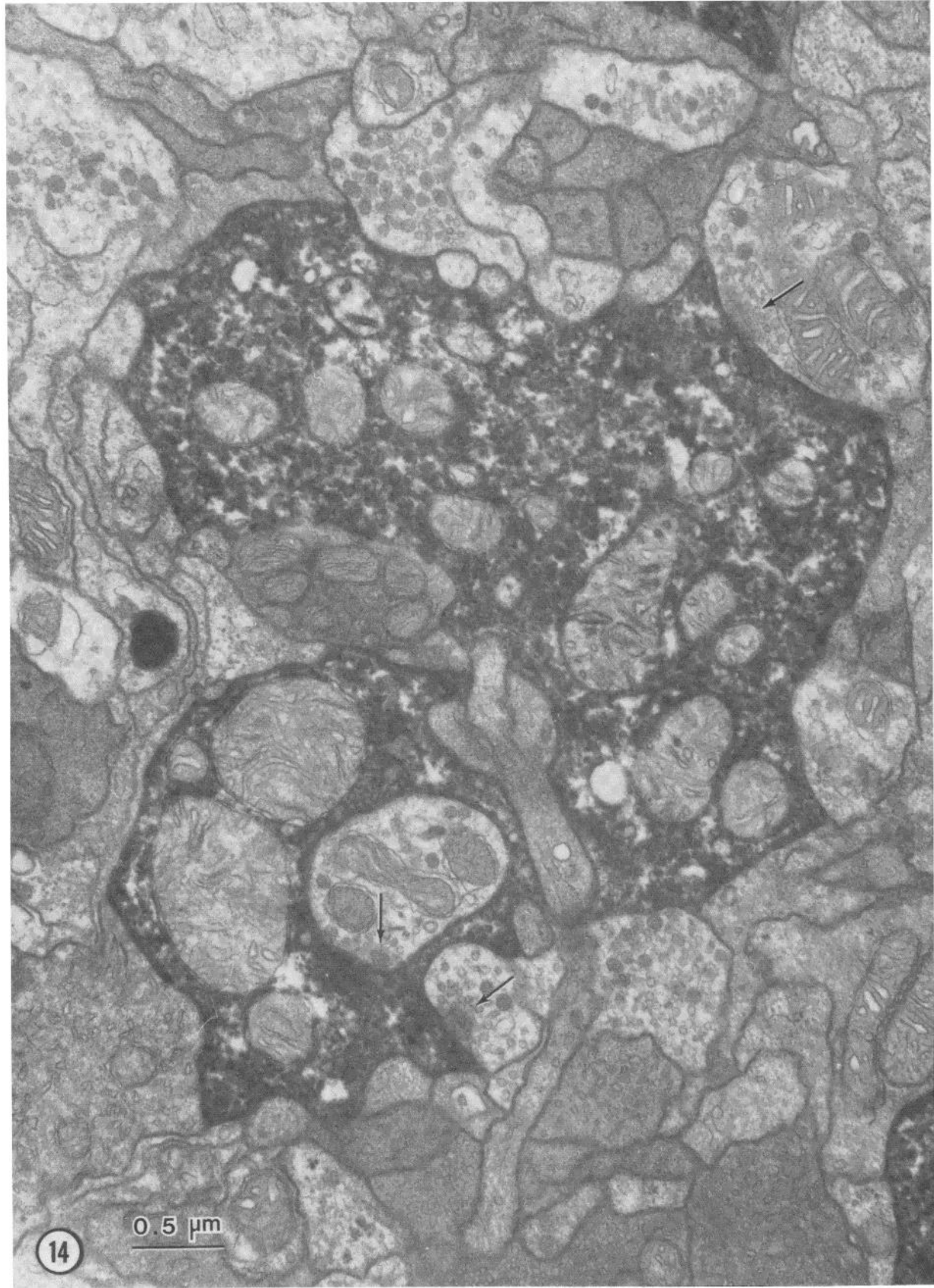

Figure 14. From specimen $P R$. An HRP-filled receptor terminal is postsynaptic to three profiles (arrows) having cytological features identical to the RL component in specimen A-I. The presynaptic densities are sectioned longitudinally (upper right), obliquely (bottom right), and transversely (bottom left). 


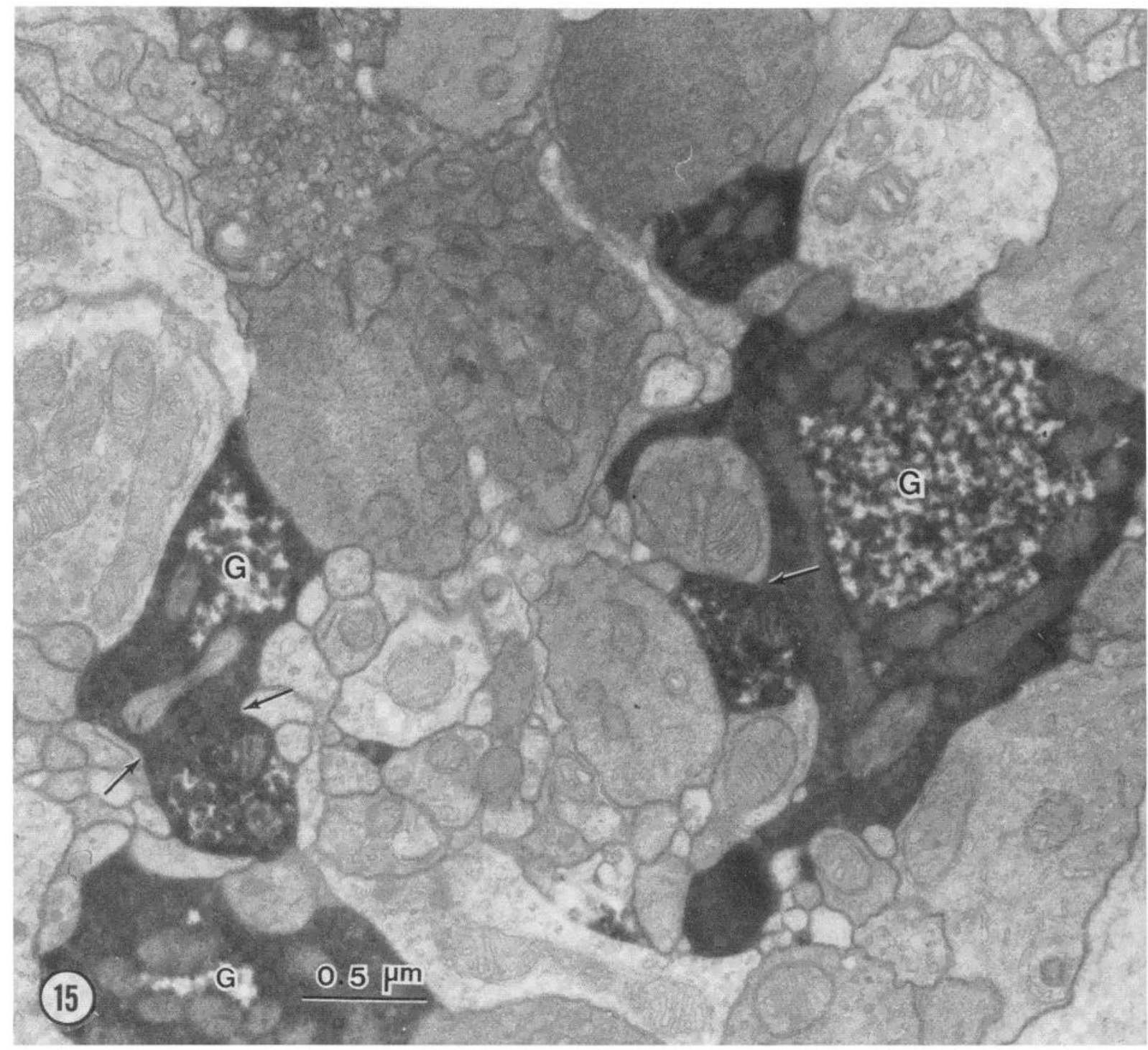

Figure 15. A section from specimen $A-I$, lateral to the photoreceptor arborization, to show that varicosities belonging to the HRP-filled I-cell are juxtaposed (at the arrows) to filled profiles of the A-cell. The arrangement of processes at the right of the figure resembles a dyad, one element of which is the HRP-filled A-cell. The identity of the cells in this region was established by tracing them to secondary processes of the I-cell and A-cell that could be distinguished by the distinctive clumping of the HRP in each cell. Note the complexity of the surrounding neuropil compared to that in Figures 8,12 , and 13 . The varicosities of the filled I-cell contain glycogen $(G)$. 


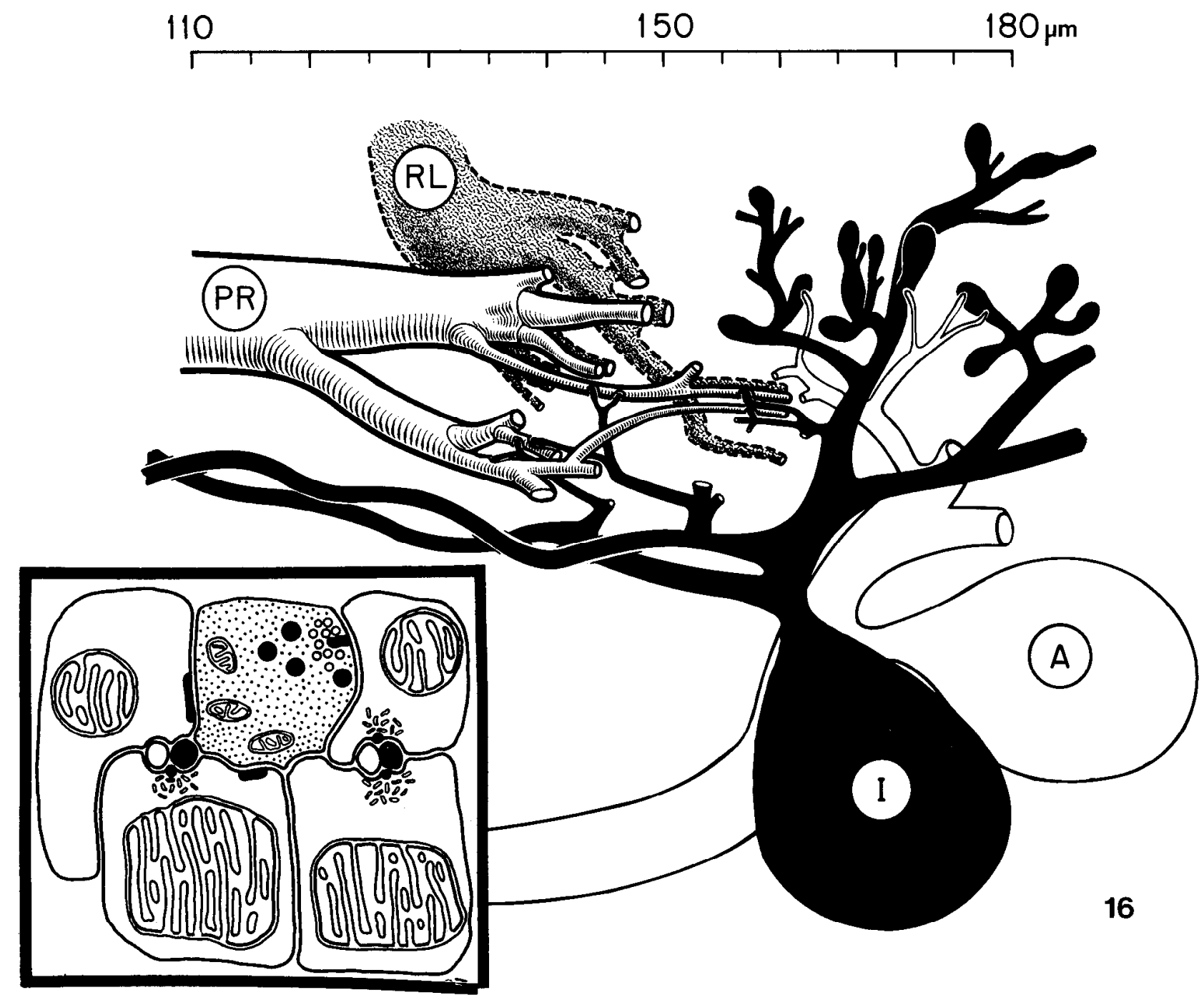

Figure 16. Schematic diagram illustrating the relationships among the branches of a median photoreceptor $(P R)$, the traced RL axon, and the injected I-cell and A-cell seen in specimen $A-I$. The following anatomical landmarks are of note: (1) the entry of the RL axons $(125$ to $135 \mu \mathrm{m}),(2)$ the most medial presynaptic sites of the photoreceptor axons (135 $\mu \mathrm{m}),(3)$ the most medial I-cell varicosities $(155 \mu \mathrm{m}),(4)$ the most medial I-cell-A-cell contacts $(160 \mu \mathrm{m})$, and (5) the end of the photoreceptor arborization $(160 \mu \mathrm{m})$. The relative spacing between these landmarks was virtually the same in specimen $P R-I$. Inset, Schematic diagram illustrating the synaptic organization in the photoreceptor-associated neuropil. Median receptor terminals (large mitochondria) are presynaptic to I-cells (solid) at dyads and make specialized junctions on RL profiles (stippled). RL profiles are presynaptic to median receptor terminals at nondyad junctions.

But as far as the median visual pathway is concerned, the I-cell to A-cell contacts are well segregated from the receptor to I-cell synapses. For the visual information, then, it seems that the complete arborization in one hemiganglion is the minimum functional unit and that postsynaptic responses must spread over significant distances to be effective in causing transmitter release from presynaptic sites onto the A-cell.

\section{References}

Graham, R. C., Jr., and M. J. Karnovsky (1966) The early stages of absorption of injected horseradish peroxidase in the proximal tubules of mouse kidney: Ultrastructure. J. Histochem. Cytochem. 14: 291-302.

Heuser, J. E., and T. S. Reese (1973) Evidence for recycling of synaptic vesicle membrane during transmitter release at the frog neuromuscular junction. J. Cell Biol. 57: 315-344.

Hudspeth, A. J., and A. E. Stuart (1977) Morphology and responses to light of the somata, axons, and terminal regions of individual photoreceptors of the giant barnacle. J. Physiol. (Lond.) 272: 1-23.

King, D. G. (1976) Organization of crustacean neuropil. I. Patterns of synaptic connections in lobster stomatogastric ganglion. J. Neurocytol. 5: 207-237.

Muller, K. J., and U. J. McMahan (1976) The shapes of sensory and motor neurons and the distribution of their synapses in ganglia of the leech: A study using intracellular injection of horseradish peroxidase. Proc. R. Soc. Lond. (Biol.) 194: 481499.

Oertel, D., and A. E. Stuart (1981) Transformation of signals by interneurones in the barnacle's visual pathway. J. Physiol (Lond.) 311: 127-146.

Oland, L. A., J. H. Hayashi, K. A. French, and A. E. Stuart (1983) The lateral visual pathway of the giant barnacle. J. Neurophysiol. 47: 516-527.

Ozawa, S., S. Hagiwara, K. Nicolaysen, and A. E. Stuart (1975) Signal transmission from photoreceptors to ganglion cells in 
the visual system of the giant barnacle. Cold Spring Harbor Symp. Quant. Biol. 40: 536-570.

Ozawa, S., S. Hagiwara, and K. Nicolaysen (1977) Neural organization of the shadow reflex in a giant barnacle, Balanus nubilus. J. Neurophysiol. 40: 982-995.

Schabtach, E., and T. A. Parkening (1974) A method for sequential high resolution light and electron microscopy of selected areas of the same material. J. Cell Biol. 61: 261-264. Schaeffer, S. F., and E. Raviola (1978) Membrane recycling in the cone cell endings of the turtle retina. J. Cell Biol. 79: 802825.

Simionescu, H., and M. Simionescu (1976) Galloylglucoses of low molecular weight as mordant in electron microscopy. I. Procedure, and evidence for mordanting effect. J. Cell Biol. 70: 608-621.

Stuart, A. E., and D. Oertel (1978) Neuronal properties underlying processing of visual information in the barnacle. Nature 275: $287-290$. 